\title{
Negatively controlled, randomized clinical trial to evaluate use of intramammary ceftiofur for treatment of nonsevere culture-negative clinical mastitis
}

\author{
M. J. Fuenzalida ${ }^{1}$ and P. L. Ruegg ${ }^{2 *}$ \\ ${ }^{1}$ Department of Dairy Science, University of Wisconsin, Madison 53706 \\ ${ }^{2}$ Department of Animal Science, Michigan State University, East Lansing 48864
}

\section{ABSTRACT}

The objective of this negatively controlled randomized clinical trial was to compare clinical outcomes of 5-d intramammary treatment using ceftiofur hydrochloride and no antimicrobial treatment of nonsevere culture-negative cases of clinical mastitis $(\mathrm{CM})$. A total of 121 cases of nonsevere (abnormal milk or abnormal milk and udder) culture-negative CM were randomly assigned to either treatment $(\mathrm{n}=62)$ or negative control $(\mathrm{n}=59)$ groups. Quarters assigned to treatment received 1 daily intramammary infusion with an approved commercially available product containing ceftiofur hydrochloride for $5 \mathrm{~d}$. Quarters assigned to the negative control group did not receive any interventions. Enrolled cows were followed for $90 \mathrm{~d}$ or until the end of lactation. At enrollment, milk samples from the affected quarter were used for on-farm culture, somatic cell count (SCC) analysis, and further microbiological analysis. During the follow-up period, milk samples were collected for microbiological analysis and SCC analysis. No significant differences between treatment and negative control groups were identified for treatment failure ( $5 \%$ for treatment vs. $10 \%$ for negative control, $\mathrm{n}=121$ ), quarter-level CM recurrence (8 vs. $5 \%, \mathrm{n}=91)$, intramammary infection at 14 or $28 \mathrm{~d}$ after enrollment ( 13 vs. $26 \%, \mathrm{n}=86$ ), days until clinical cure ( 4.2 vs. 4.0 d), days to culling ( 48.3 vs. 36.8 d), daily milk production (43.3 vs. $43.6 \mathrm{~kg} / \mathrm{cow}$ per day), or weekly quarter SCC (5.5 vs. $5.4 \log _{10}$ SCC). Days of milk discard were greater for cows assigned to the treatment group $(8.5 \mathrm{~d})$ compared with cows assigned to the negative control group ( $5.6 \mathrm{~d})$. During the follow-up period, cases in the treatment group had a $50 \%$ risk reduction in IMI compared with cases in the negative control group. Irrespective of group, negative

Received August 3, 2018.

Accepted November 29, 2018

*Corresponding author: plruegg@msu.edu outcomes such as quarter-level CM recurrence (12\%), treatment failure $(12 \%)$, and culling $(5 \%)$ occurred infrequently in nonsevere culture-negative cases of CM. Use of intramammary ceftiofur for treatment of nonsevere culture-negative cases of $\mathrm{CM}$ did not improve any economically relevant clinical outcome such as culling, milk production, or SCC.

Key words: clinical trial, clinical mastitis, dairy, antimicrobial, mastitis

\section{INTRODUCTION}

Mastitis is the most widely diagnosed disease on US dairy farms. The most common strategy for dealing with clinical mastitis $(\mathbf{C M})$ is to administer intramammary (IMM) or systemic antimicrobials (USDA, 2014). Increased societal concerns about usage of antimicrobials in farm animals and the growing crisis of antimicrobial resistance (Sifferlin, 2017) have created challenges for continued use of antimicrobials in farm animals. Strong evidence of increased resistance of mastitis pathogens is lacking (Erskine et al., 2002; Makovec and Ruegg, 2003a); however, it is important to ensure that antimicrobials are used appropriately and sparingly. Although antimicrobial usage is best reduced by preventing mastitis, after detection of CM, antimicrobial usage can be reduced by understanding the distribution of pathogens causing CM and applying selective therapy to cows that are affected with pathogens that will benefit from use of antimicrobials (Pinzón-Sánchez and Ruegg, 2011; Oliveira et al., 2013; Oliveira and Ruegg, 2014).

Clinical mastitis is often categorized based on the magnitude of inflammation. About $85 \%$ of cases are categorized as mild (abnormal milk) or moderate (abnormal milk and abnormal udder), which together are referred to as nonsevere (Oliveira et al., 2013). In nonsevere cases of CM, inflammation is localized within the mammary gland. In the United States, the majority of mastitis treatments used for this category are administered as IMM preparations (USDA, 2014). On modern US dairy farms, microbiological examination 
of milk collected from cases of CM usually results in approximately one-third gram-negative bacteria, onethird gram-positive bacteria, one-third culture-negative milk samples, and $10 \%$ other pathogens (Oliveira et al., 2013). In larger confined dairy herds, the most prevalent mastitis pathogens include Escherichia coli (22.5\%) and environmental streptococci (12.8\%; Oliveira et al., 2013). Researchers have suggested that antimicrobials are not necessary for treatment of mastitis caused by E. coli (Pyörälä et al., 1994; Pyörälä and Pyörälä, 1998) or microbiologically negative cases (Oliveira and Ruegg, 2014). In contrast, antimicrobial therapy is generally considered beneficial for mastitis caused by environmental streptococci (Oliver et al., 2004a,b). As herd sizes have increased and management has intensified, the proportion of culture-negative cases of $\mathrm{CM}$ has increased due to success in control of contagious pathogens and increased exposure to environmental pathogens (Makovec and Ruegg, 2003b). Culture-based selective treatment protocols are based on targeting antimicrobial usage for culture-positive cases while allowing for spontaneous cure of culture-negative and gram-negative cases, and use of these protocols has been demonstrated to reduce use of antimicrobials for treatment of mastitis (Lago et al., 2011a,b; Vasquez et al., 2017). Positively controlled randomized clinical trials (Sérieys et al., 2005; Swinkels et al., 2013, 2014; Mansion-de Vries et al., 2016) and observational studies (Pyörälä and Pyörälä, 1998; Pinzón-Sánchez and Ruegg, 2011; Oliveira and Ruegg, 2014) have described pathogen-specific outcomes of various antimicrobial treatments for nonsevere CM, but few negatively controlled randomized clinical trials have been published. The objective of this negatively controlled randomized clinical trial was to compare clinical outcomes of 5 -d IMM treatment using ceftiofur hydrochloride with no antimicrobial treatment of nonsevere culture-negative cases of CM.

\section{MATERIALS AND METHODS}

\section{Study Design}

A negatively controlled, randomized clinical trial was conducted using dairy cattle housed at the University of Wisconsin ( UW) dairy farms. Lactating cows were managed as a single herd but were housed at 2 locations, with some cows transferred between locations based on research and teaching needs. The largest facility housed approximately 470 lactating dairy cows in sand-bedded freestall barns and the smallest facility housed approximately 66 lactating dairy cows in a tie-stall barn. In both locations, lactating cows were milked twice daily in parallel milking parlors by trained milking technicians using standardized milking practices that included removal and observation of foremilk.

Cows were enrolled between March 2015 and May 2017. The herd had monthly DHIA testing that included individual cow SCC data, used computerized herd records (including recording of mastitis events and daily milk production), and administered internal teat sealants (Orbeseal, Zoetis, Parsippany-Troy Hills, NJ) and IMM dry cow therapy (Quartermaster, Zoetis) to all cows at the end of every lactation. Before the study began, farm personnel were trained to aseptically collect quarter milk samples, record a standardized mastitis severity score (Pinzón-Sánchez and Ruegg, 2011), and perform on-farm culture (OFC; Lago et al., 2011a).

\section{Case Enrollment Criteria}

Upon detection of CM, farm workers assigned a severity score that was used to determine eligibility for enrollment. Only cows with nonsevere cases were eligible for screening for inclusion in the study. Cows with severe cases (abnormal milk accompanied by systemic signs) were treated immediately and were not eligible. Initially, based on concerns of farm managers, daily milk production was also used as a criterion for study enrollment. During March to July 2015, cows experiencing a more than $20 \%$ reduction in milk yield (in addition to signs of nonsevere CM) were excluded; however, this criterion was removed in August 2015.

Milking technicians aseptically collected duplicate quarter milk samples from eligible nonsevere cases of CM. One sample was used for performing on-farm culture by using a swab containing approximately 100 $\mu \mathrm{L}$ of milk to inoculate an agar biplate that contained MacConkey agar (selective for gram-negative bacteria) on one half and a proprietary medium selective for gram-positive bacteria (MN Biplate, Minnesota Easy Culture System, University of Minnesota Laboratory for Udder Health, 2004) on the other half. The remaining milk in that sample was preserved with bronopol for SCC analysis. The duplicate milk sample was frozen for further microbiological testing at the UW Milk Quality laboratory. Twenty-four hours after inoculation of the OFC biplates, trained farm workers observed plates for microbial growth. Culture-positive cases were not enrolled and were treated according to the farm protocol (Figure 1). Culture-negative cases were randomly assigned to either the treatment or negative control group (Figure 1). Random allocation of cases was performed by opening envelopes that contained the assignment. Study personnel retrieved envelopes every week to confirm compliance with the allocation protocol.

If more than 1 quarter was affected with nonsevere $\mathrm{CM}$, all quarters were sampled and cultured. If all 
quarters were culture negative, affected quarters were assigned to the same experimental groups. If culture results varied among quarters, the cow was excluded. On-farm culture was not available at the smaller facility, so farm workers contacted researchers to pick up milk samples collected from eligible cases within $2 \mathrm{~h}$ and researchers rather than farm workers performed OFC. For both locations, enrollment decisions were made based on 24-h culture results.

After enrollment, if the case worsened in severity or failed to improve within $72 \mathrm{~h}$, additional treatments were allowed. Cases that progressed to severe were treated as defined by herd protocols and were removed from the study and categorized as treatment failures (TF). When recurrent cases of CM were detected in the same enrolled quarter or a different quarter, OFC was performed. When the case was culture negative, it was assigned to the same experimental group as previously assigned. All procedures were approved by the Animal Care and Use Committee for the College of Agriculture and Life Sciences of the UW Madison (protocol A01574-0-06-14).

\section{Interventions}

Quarters assigned to treatment received 1 daily IMM infusion with a commercially available approved product containing ceftiofur hydrochloride (Spectramast $\mathrm{LC}$, Zoetis) for $5 \mathrm{~d}$. Quarters assigned to the negative control group did not receive any antimicrobial therapy

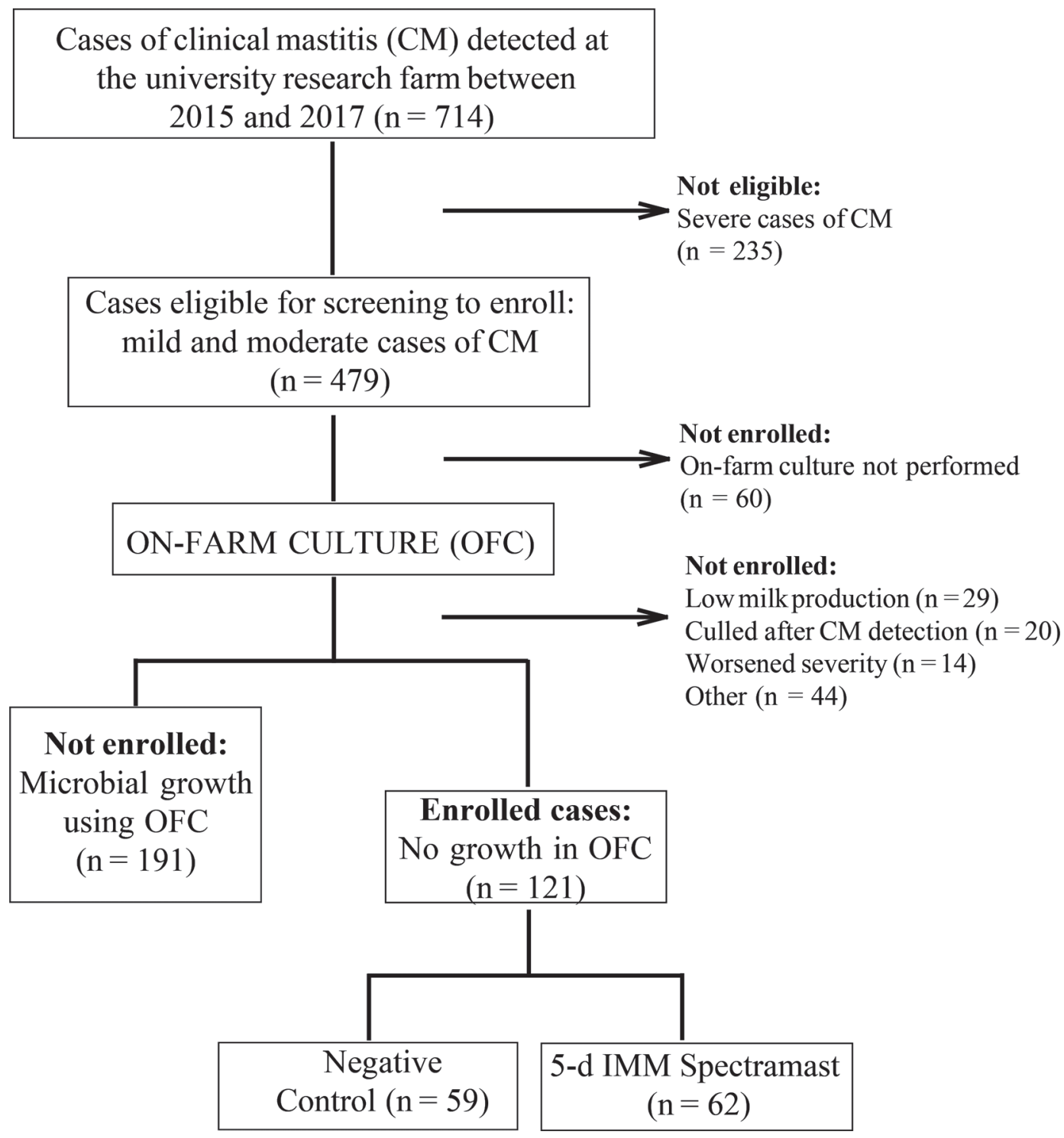

Figure 1. Flow diagram for enrollment of cases of clinical mastitis in the study. Reasons for exclusion and nonenrollment in the study are depicted. Spectramast LC is manufactured by Zoetis (Parsippany-Troy Hills, NJ). 
or any other interventions. Cows enrolled in both groups were moved to the hospital pen and milk was discarded until it returned to normal or until the end of the milk-withholding period for ceftiofur.

\section{Data Collection}

Data were collected for a 90-d follow-up period (FUP) or until the end of lactation. For the first 10 d after enrollment, at each milking farm workers recorded appearance of milk and udder and evaluated cow attitude. During the FUP, farm workers aseptically collected quarter milk samples and conducted OFC from quarters that experienced recurrent CM. Researchers visited farms once weekly to retrieve cow records; aseptically collect duplicate milk samples from enrolled quarters at approximately $7,14,28,63$, and $84 \pm 3 \mathrm{~d}$ after enrollment; and assess compliance. One duplicate milk sample collected during the FUP was used for microbiological analysis, and the other sample was preserved with bronopol and transported to a commercial DHIA laboratory (AgSource CRI, Verona, WI) for SCC analysis.

\section{Microbiological Analysis}

Microbiological analysis of quarter milk samples collected at d $0,7,14,28,63$, and $84 \pm 3$ was performed at the UW Milk Quality laboratory according to National Mastitis Council guidelines (NMC, 1999, 2017). Frozen milk samples collected by farm personnel were thawed at room temperature, and fresh milk samples were plated upon arrival at the laboratory. One-hundred microliters of milk was inoculated onto half of a blood agar plate (Trypticase soy agar with $5 \%$ sheep blood; BD Diagnostic Systems, Becton Dickinson, Franklin Lakes, NJ). An additional $10 \mu \mathrm{L}$ of milk was inoculated onto one-quarter of a MacConkey plate. Plates were incubated at $37^{\circ} \mathrm{C}$ for 24 to $48 \mathrm{~h}$. Gram staining characteristics were described for all isolates. To differentiate among gram-negative bacteria, isolates that grew on MacConkey agar were inoculated on triple sugar iron slants, motility, indole and ornithine, and sodium citrate slants. For gram-positive bacteria, catalase tests were performed to differentiate Staphylococcus and Streptococcus. Mannitol and tube coagulase reactions were performed for catalase-positive colonies. Christie, Atkins, Munch-Petersen, esculin, and bile esculin reactions were performed for catalase-negative colonies. Gram staining characteristics, colony morphology, catalase reactions (Christie, Atkins, Munch-Petersen, esculin, and bile esculin or coagulase and mannitol reactions) and biochemical tests were described to arrive at a final microbiological diagnosis. Mixed infection was defined as at least 10 colonies of 2 types of bacteria found in the same milk sample. Milk samples were considered contaminated when 3 or more colony types were isolated from the same sample. Culture-negative milk samples were defined as milk samples that did not result in growth of any bacteria on blood or MacConkey agar. Nonsignificant growth was defined as growth of less than $100 \mathrm{cfu} / \mathrm{mL}$ of 1 or 2 types of bacteria from a single milk sample (Andersen et al., 2010; PinzónSánchez and Ruegg, 2011). For analysis, results of quarter milk samples with nonsignificant growth were combined with no growth. Plates were evaluated for bacterial growth at 24 and $48 \mathrm{~h}$ after incubation. Final microbiological results of samples processed at the UW Milk Quality Laboratory were based on the 48-h evaluation. For milk samples collected at d 0, an IMI was defined as the isolation of $100 \mathrm{cfu} / \mathrm{mL}$ of identical colonies except for Staphylococcus aureus, whereby an IMI was defined as the isolation of at least $10 \mathrm{cfu} /$ $\mathrm{mL}$. During the FUP, an IMI was defined as described by Dohoo et al. (2011) for multiple consecutive milk samples (briefly, the isolation of the same pathogen in 2 or more consecutive follow-up samples or the isolation of a pathogen with more than $1,000 \mathrm{cfu} / \mathrm{mL}$ once during the FUP).

\section{Definitions}

Cases of CM that occurred at least $14 \mathrm{~d}$ after a previous case were considered new cases. Subclinical mastitis (SCM) was defined when monthly DHIA SCC exceeded 150,000 cells/mL (Lavon et al., 2011).

Risk Factors. Clinical mastitis history was defined as occurrence of a CM event during the $55 \mathrm{~d}$ before case detection. Previous disease was defined as occurrence of a disease (i.e., ketosis, retained placenta, milk fever, pneumonia, and metritis) before detection of the enrolled case.

Outcomes. Intramammary infection at 14 or $28 \mathrm{~d}$ after enrollment was defined as isolation of bacteria from quarter milk samples at either period (Dohoo et al., 2011). Treatment failure was defined as administration of a treatment different from the assigned antimicrobial treatment due to worsening severity of CM, continued abnormal appearance of milk for $10 \mathrm{~d}$ after enrollment, or recurrence of abnormal milk in the same quarter after it had earlier returned to normal (within $14 \mathrm{~d}$ after enrollment). Days until clinical cure was the number of days until the milk returned to visually normal appearance for at least 2 consecutive days (4 consecutive milkings) within $10 \mathrm{~d}$ after enrollment. Recurrence of $\mathrm{CM}$ was assessed only in cases that achieved clinical cure. Quarter-level CM recurrence (QR) during the FUP was defined as detection of abnormal milk in the 
same quarter more than14 d after enrollment. Days of milk discard was defined as the number of days the milk was not eligible for sale due to abnormal appearance, treatment, or the withholding period. Days until IMI was the number of days until isolation of bacteria from quarter milk samples during the FUP. Culling was defined as removal during the FUP due to sale. Daily milk production was automatically collected from milk meters.

\section{Statistical Analysis}

Statistical analyses were performed using SAS version 9.4 (SAS Institute, 2011). The experimental unit was quarter, and analyses followed intention-to-treat principles. Binary categorical outcomes were TF, QR, and IMI at 14 or $28 \mathrm{~d}$ after enrollment. Continuous outcomes were daily milk yield and weekly quarter SCC (QSCC) during the FUP, and time-to-event outcomes included days to clinical cure, days of milk discard, days until IMI, and culling. Categorical explanatory variables were parity group ( 1 and 2,3 and 4 , and $>4$ ), severity of CM (mild or moderate), occurrence of previous diseases (yes or no), CM history (yes or no), and season of enrollment (fall, winter, summer, spring). Subclinical mastitis history was assessed as a dichotomous variable (previous SCM; yes or no) or continuous variable (previous SCC 21 to $55 \mathrm{~d}$ before case detection). Continuous variables included average daily milk yield ( $\mathrm{kg} / \mathrm{cow}$ per day) from 1 to $7 \mathrm{~d}$ before case detection and DIM. Somatic cell count data were log transformed $\left(\log _{10} \mathrm{SCC}=\log _{10} \mathrm{SCC} \times 1,000\right)$ to follow normal distribution.

We used PROC FREQ to describe the distribution of cases of CM that occurred during the enrollment period and characteristics of cases enrolled in the study. For categorical variables, comparisons were made using chi-squared or Fisher's exact tests. For continuous variables, differences in variables were calculated using ANOVA tests with PROC GLM. Univariate associations between categorical outcomes and selected risk factors were evaluated using chi-squared or Fisher exact tests with PROC FREQ. Variables with a $P$-value $<0.25$ using univariate analyses were eligible to be offered to the multivariate analyses.

To analyze agreement between the microbiological results of the UW Milk Quality Laboratory and OFC, the kappa statistic (Cohen, 1960) was calculated using PROC FREQ. The null hypothesis was that agreement between OFC and the UW Milk Quality Laboratory was due to chance. Microbiological categories were gram positive, gram negative, no growth, and contaminated.

Number needed to treat was calculated to describe the effect of the 5-d ceftiofur IMM therapy compared with no treatment (Altman and Andersen, 1999). Power was calculated using a $0.05 \alpha$-significance level, the number of events, and proportion of the outcome for treatment and control groups. Survival probabilities were calculated for clinical cure, days of milk discard, culling, and IMI by experimental group and selected risk factors using PROC LIFETEST. Time corresponded to days until the event of interest occurred (response variable). Cases were censored when the information about their survival was not available due to right censoring (the event never occurred during the FUP) or lost to followup. The Kaplan-Meier method was used to calculate survival probabilities (log rank test). We used PROC PHREG to estimate the hazard ratio at which time-toevent outcomes occurred during the FUP while adjusting for different covariates. The ASSESS statement was used to analyze the proportional hazards assumption.

Multivariate logistic regression models were performed using PROC GLIMMIX. Selected risk factors associated with clinical and treatment outcomes were offered to the model. The effect of treatment was included in all models. Goodness of fit of each model was assessed using the $-2 \log$ likelihood, Akaike information criterion (AIC), small-sample-corrected AIC (AICC), and Bayesian information criterion of PROC GLIMMIX. Parity group was offered to the model as a 2 - (1 or 2 parity and $>2$ parities) or 3 - (as previously described) level category based on the goodness of fit for each model. Subclinical mastitis was offered as a categorical or continuous variable based on goodness of fit. Each variable offered to the model was evaluated by manual bidirectional elimination until all explanatory variables had $P$-value $<0.10$; subsequently, a model was chosen based on parsimony and goodness of fit. Adjusted probabilities of clinical and treatment outcomes were generated using the LSMEANS and ODDS RATIO option via PROC GLIMMIX. Cases were not included in an analysis if they had missing data for a variable included in a model.

Model 1 used data from 91 cases to assess the effect of 5 -d IMM ceftiofur therapy of nonsevere culture-negative $\mathrm{CM}$ on $\mathrm{QR}$. The null hypothesis was that there is no difference in the probability of QR between treated and negative control cases. After model building, remaining explanatory variables included treatment (forced), average daily milk yield 1 to $7 \mathrm{~d}$ before case detection, and $\log _{10}$ SCC 21 to $55 \mathrm{~d}$ before case detection. Model 2 used data from 86 cases to assess the effect of treatment on IMI at 14 or $28 \mathrm{~d}$ after enrollment. The null hypothesis was that there is no difference in the probability of IMI between treated and negative control cases. After model building, remaining explanatory variables included treatment (forced), parity group, and $\log _{10}$ SCC 21 to $55 \mathrm{~d}$ before case detection. Model 
3 used data from 121 cases to assess the effect of 5 -d IMM ceftiofur therapy of nonsevere culture-negative $\mathrm{CM}$ on TF. The null hypothesis was that there is no difference in the probability of TF between treated and negative control cases. After model building, remaining explanatory variables included treatment (forced), parity group, and DIM.

To analyze the effect of IMM treatment on daily milk production and weekly QSCC, repeated-measures analysis of ANOVA was conducted using PROC MIXED with autoregressive and unstructured covariance structures, respectively. After model building, for daily milk production, remaining explanatory variables included time, treatment (forced), parity group, season of enrollment, DIM, average daily milk production 1 to $7 \mathrm{~d}$ before case detection, and 2-way interaction. The null hypothesis was no difference in daily milk production between treated and negative control cases. After model building, for weekly QSCC, remaining explanatory variables included time, treatment (forced), parity group, CM history, previous SCM, occurrence of IMI during FUP, and 2-way interaction. The null hypothesis was no difference in weekly QSCC between treated and negative control cases. After model building, for the association of IMI with daily milk production, remaining explanatory variables included time, occurrence of IMI during FUP, season of enrollment, DIM, average daily milk production 1 to $7 \mathrm{~d}$ before case detection, previous SCM, and 2-way interaction. The null hypothesis was no difference in daily milk production between cases experiencing IMI and not experiencing IMI.

\section{RESULTS}

\section{Characteristics of Herd and Cases of CM}

During the enrollment period, the UW Madison research dairy farm comprised about 530 dairy cows, with approximately $88 \%$ housed at the larger freestall facility. Parity group distribution did not vary among years $(P=0.903)$. Average milk production and individual cow SCC during the enrollment period were about 42 $\mathrm{kg} /$ cow per day and 63,095 cells $/ \mathrm{mL}$, respectively. The incidence of $\mathrm{CM}$ ranged from 2.6 to $3.8 \mathrm{CM}$ cases/100 cows per month (Table 1).

Characteristics of cases of CM did not vary by year $(P<0.05$; Table 1$)$. Of cases of $\mathrm{CM}$ detected during the enrollment period, the distribution of severity was approximately $70 \%$ nonsevere (mild or moderate) and $30 \%$ severe. Overall, more than $90 \%$ of the cases of $\mathrm{CM}$ occurred in a single quarter. Among enrolled cases $(\mathrm{n}=121)$, there was an equal proportion of mild and moderate cases.

\section{Enrollment}

During the enrollment period, farm workers detected 714 cases of CM, of which 479 were eligible for screening (Figure 1). During this period, the overall distribution of microbiological results of CM cases (based on routine culture at the UW Milk Quality Laboratory) was approximately $20 \%$ gram negative, $43 \%$ culture negative, $3 \%$ Staph. aureus, $6 \%$ NAS, $24 \%$ environmental streptococci and Enterococci spp., and $4 \%$ other pathogens. Of potentially eligible cases, culture was not performed in $8.1 \%(33 / 407)$ and $37.5 \%(27 / 72)$ of cases detected while cows were housed in the larger and smaller facilities, respectively. For cows at the larger facility, microbial growth in OFC was the most common reason for not enrolling. We enrolled 119 cases of CM affecting a single quarter and 2 cases of CM from 2 cows affected with CM in 2 quarters. In each cow with multiple quarters, only 1 randomly selected quarter was enrolled. Of 121 enrolled cases, 112 came from cows located in the larger facility at the time of detection of $\mathrm{CM}$ and 9 came from cows located at the smaller facility at the time of detection of CM. After randomization, $51 \%$ of cases were enrolled in the treated group, whereas $49 \%$ were enrolled in the control group (Figure 1).

\section{Agreement Between OFC and UW Milk Quality Laboratory Results}

Observed agreement between results of OFC and the UW Milk Quality Laboratory was 60 to $64 \%$, and kappa values were moderate $(\mathrm{Z}=10.7, P<0.001$; Cohen, 1960). Disagreement between the OFC and UW Milk Quality Laboratory results was categorized as follows: bacterial growth in the UW Milk Quality Laboratory ( $7.6 \%$ at $24 \mathrm{~h}$ and $12.1 \%$ at $48 \mathrm{~h}$ ) but culture-negative results of OFC; different OFC diagnosis compared with result of the UW Milk Quality Laboratory $(2.5 \%$ at 24 or $48 \mathrm{~h}$ ); bacterial growth from OFC for culture-negative results in the UW Milk Quality Laboratory (18.1\% at $24 \mathrm{~h}$ and $14.9 \%$ at $48 \mathrm{~h}$ ); and bacterial growth from OFC but contaminated results in the UW Milk Quality Laboratory ( $7.6 \%$ at $24 \mathrm{~h}$ and $10.0 \%$ at $48 \mathrm{~h}$ ).

\section{Description of Experimental Groups}

The distribution of parity group, severity of CM, year and season of enrollment, previous disease, and CM history did not vary based on experimental group (Table 2). Days in milk at case detection were $159.9 \pm$ 12.6 and $134.6 \pm 11.7$ for cows assigned to the negative control and treated groups, respectively $(P=0.144)$. Average daily milk production from 1 to $7 \mathrm{~d}$ before case 
Table 1. Characteristics of herd and overall cases of clinical mastitis (CM) at the University of Wisconsin research and teaching farm

\begin{tabular}{|c|c|c|c|c|c|c|c|c|}
\hline Characteristic & \multicolumn{6}{|c|}{ Year } & $P$-value ${ }^{1}$ & Total \\
\hline Parity of cows & & & & & & & 0.903 & \\
\hline 1 & 23.0 & 123 & 23.4 & 123 & 22.6 & 122 & & \\
\hline 2 & 32.1 & 171 & 33.2 & 174 & 31.1 & 167 & & \\
\hline Individual $\log _{10} \mathrm{SCC}^{2,3}$ & & $4.8 \pm 0.0$ & & $4.8 \pm 0.0$ & & $4.9 \pm 0.0$ & - & - \\
\hline Monthly DHIA milk yield, ${ }^{2,3} \mathrm{~kg} / \mathrm{cow}$ & & $43.6 \pm 0.1$ & & $41.2 \pm 0.1$ & & $43.8 \pm 0.1$ & - & - \\
\hline Incidence of $\mathrm{CM}^{2,4}$ & & $3.5 \pm 0.4$ & & $3.8 \pm 0.4$ & & $2.6 \pm 0.4$ & - & - \\
\hline Total cases of $\mathrm{CM},{ }^{5}$ no. & & 276 & & 346 & & 92 & & 714 \\
\hline Severity of $\mathrm{CM}^{6}$ & & & & & & & 0.748 & \\
\hline Nonsevere & 66.3 & 183 & 66.8 & 231 & 70.6 & 65 & & 479 \\
\hline Severe & 33.7 & 93 & 33.2 & 115 & 29.4 & 27 & & 235 \\
\hline Severity of CM & & & & & & & 0.621 & \\
\hline Mild & 50.0 & 20 & 55.4 & 36 & 62.5 & 10 & & 66 \\
\hline Moderate & 50.0 & 20 & 44.6 & 29 & 37.5 & 6 & & 55 \\
\hline
\end{tabular}

${ }^{1}$ Comparisons between year and severity of CM or number of quarters affected with CM were performed using chi-squared analysis or Fisher's exact test.

${ }^{2}$ Mean \pm SEM.

${ }^{3}$ Herd average by year was calculated from monthly DHIA test data (individual milk yield and SCC).

${ }^{4}$ Number of CM cases per 100 cows per month. The incidence of CM is the number of new cases of CM divided by the number of lactating cows at risk (lactating cows that have not been diagnosed with CM) per month per year. Cases of CM that occurred $\geq 14 \mathrm{~d}$ from previous cases were considered new cases.

${ }^{5}$ Total cases of CM were counted March through December 2015, January through December 2016, and January through May 2017. ${ }^{6}$ Mild or moderate cases of CM were considered nonsevere. Severe CM was defined as abnormal milk accompanied by systemic signs.

${ }^{7}$ Number of quarters affected with CM was either single (only 1 quarter affected with $\mathrm{CM}$ ) or multiple (>1 quarter affected with CM).

${ }^{8}$ Cows were enrolled March 2015 through May 2017.

detection was $45.2 \pm 1.1$ and $47.4 \pm 1.1 \mathrm{~kg}$ for cows assigned to the negative control and treatment groups, respectively $(P=0.186)$. Average $\log _{10}$ SCC 21 to 55 $\mathrm{d}$ before case detection was $5.0 \pm 0.1$ and $5.1 \pm 0.1$ $\log _{10}$ SCC for cows assigned to the negative control or treated groups, respectively $(P=0.435)$.

\section{Quarter-Level Recurrence}

Overall, $12 \%$ of enrolled cows developed QR within the FUP (Table 3). Of categorical variables evaluated, experimental group, parity group, and CM history met criteria for entry into multivariate models. Milk production 1 to $7 \mathrm{~d}$ before case detection was $53.9 \pm 2.4$ and $45.6 \pm 0.9 \mathrm{~kg}$ for cows experiencing $\mathrm{QR}$ and cows not experiencing $\mathrm{QR}$, respectively $(P=0.001)$. $\log _{10}$ SCC 21 to $55 \mathrm{~d}$ before the case detection was $5.5 \pm 1.2$ and $5.0 \pm 0.1 \log _{10}$ SCC for cows experiencing a recurrent case and cows not experiencing QR, respectively $(P=0.012)$.

After model fitting, the final logistic regression model for effect of IMM treatment of culture-negative nonse- vere $\mathrm{CM}$ on $\mathrm{QR}$ included treatment (forced), average milk yield 1 to $7 \mathrm{~d}$ before the case detection, and $\log _{10}$ SCC 21 to 55 d before the case detection (Table 4). Compared with cows in the negative control group, IMM treatment did not have an effect on probability of QR $(P=0.524)$. For every kilogram of milk produced 1 to $7 \mathrm{~d}$ before the case detection, the probability of quarter-level recurrence increased by $16 \%(P=0.003$; Table 4). For every $1 \log _{10}$ SCC unit increase 21 to 55 $\mathrm{d}$ before the case detection, a case was 1.5 times more likely to experience QR $(P=0.009$; Table 4$)$.

The power to detect a difference in $\mathrm{QR}$ by experimental group was 0.19. If the true rates of QR in the negative control and treated groups were 8.0 and $15.8 \%$ (based on distribution described in Table 3), respectively, 300 cases per experimental group would be required to reject the null hypothesis (power, $P=0.8$ ).

\section{IMI at 14 or $28 d$}

Of enrolled cows, $23 \%$ developed an IMI within 14 or 28 d. Most IMI were caused by gram-positive bacteria 
$(78 \%, \mathrm{n}=31)$, and most IMI were identified by $14 \mathrm{~d}$ postenrollment (73\%; Table 5). The most commonly isolated pathogens were environmental streptococci $(35 \%)$ and NAS (20\%). Occurrence of IMI at 14 or $28 \mathrm{~d}$ was associated with previous SCC (21-55 d before case detection; $P=0.014$; Table 6 ).

Of the categorical variables evaluated, experimental group, parity group, and CM history met the criteria for entry into multivariate models. $\log _{10}$ SCC 21 to 55 $\mathrm{d}$ before case detection was $5.4 \pm 0.1$ and $4.9 \pm 0.1 \log _{10}$ SCC for cows that experienced or did not experience IMI at 14 or $28 \mathrm{~d}$ after enrollment, respectively $(P=$ 0.008 ).

After model fitting, the final logistic regression model for effect of IMM treatment of culture-negative nonsevere $\mathrm{CM}$ on IMI at 14 or $28 \mathrm{~d}$ after enrollment included treatment (forced), parity group, and $\log _{10}$ SCC 21 to $55 \mathrm{~d}$ before case detection (Table 4). Compared with cows in the negative control group, IMM treatment did not have an effect on IMI at 14 or $28 \mathrm{~d}$ after enrollment $(P=0.165)$. Compared with cows in first or second lactation, cows in third or fourth lactation were 7 times more likely to experience IMI at 14 or $28 \mathrm{~d}$ after enroll- ment $(P=0.009)$. For every $1 \log _{10}$ SCC unit increase 21 to $55 \mathrm{~d}$ before case detection, a cow was 1.1 times more likely to experience IMI in the FUP $(P=0.018$; Table 4).

The power to detect a difference in IMI by experimental group was 0.16 . If the true rate of IMI at 14 or $28 \mathrm{~d}$ after enrollment in the negative control and treated groups was 28.9 and $18.5 \%$ (based on distribution described in Table 3), respectively, 280 cases per experimental group would be required to reject the null hypothesis (power, $P=0.8$ ).

\section{Treatment Failure}

Overall, $12 \%$ of enrolled cows were classified as experiencing TF (Table 3). Of categorical variables evaluated, experimental group and parity group were eligible for entry into multivariate models. Days in milk at case detection were $195.8 \pm 25.1$ and $140.5 \pm 9.1$ for cows that experienced or did not experience $\mathrm{TF}$, respectively $(P=0.040$; Table 3$)$.

After model fitting, the final logistic regression model for effect of IMM treatment of culture-negative non-

Table 2. Description of cases $(n=121)$ enrolled in the study by experimental group

\begin{tabular}{|c|c|c|c|c|c|c|}
\hline \multirow[b]{3}{*}{ Factor } & \multirow{3}{*}{$\begin{array}{c}\text { Total } \\
\text { no. }\end{array}$} & \multicolumn{4}{|c|}{ Experimental group ${ }^{1}$} & \multirow[b]{3}{*}{$P$-value ${ }^{2}$} \\
\hline & & \multicolumn{2}{|c|}{$\begin{array}{l}\text { Negative control } \\
\quad(\mathrm{n}=59)\end{array}$} & \multicolumn{2}{|c|}{$\begin{array}{l}\text { Treated } \\
(\mathrm{n}=62)\end{array}$} & \\
\hline & & $\%$ & No. & $\%$ & No. & \\
\hline Severity of clinical mastitis & & & & & & 0.765 \\
\hline Mild & 66 & 55.9 & 33 & 53.3 & 33 & \\
\hline Moderate & 55 & 44.1 & 26 & 46.8 & 29 & \\
\hline Period of enrollment ${ }^{3}$ & & & & & & 0.126 \\
\hline 2015 & 40 & 35.6 & 21 & 30.6 & 19 & \\
\hline 2016 & 65 & 45.8 & 27 & 61.3 & 38 & \\
\hline 2017 & 16 & 18.6 & 11 & 8.1 & 5 & \\
\hline Season of enrollment & & & & & & 0.586 \\
\hline Winter (Dec.-Feb.) & 27 & 20.3 & 12 & 24.2 & 15 & \\
\hline Spring (Mar.-May) & 38 & 37.3 & 22 & 25.8 & 16 & \\
\hline Summer (Jun.-Aug.) & 19 & 15.3 & 9 & 16.1 & 10 & \\
\hline Fall (Sep.-Nov.) & 37 & 27.1 & 16 & 33.9 & 21 & \\
\hline Parity group & & & & & & 0.404 \\
\hline $1-2$ & 42 & 40.7 & 24 & 29.0 & 18 & \\
\hline $3-4$ & 45 & 33.9 & 20 & 40.3 & 25 & \\
\hline$\geq 5$ & 34 & 25.4 & 15 & 30.7 & 19 & \\
\hline Previous disease $^{4}$ & & & & & & 0.732 \\
\hline Yes & 22 & 17.0 & 10 & 19.4 & 12 & \\
\hline No & 99 & 83.0 & 49 & 80.6 & 50 & \\
\hline Clinical mastitis history ${ }^{5}$ & & & & & & 0.573 \\
\hline Yes & 23 & 16.9 & 10 & 21.0 & 13 & \\
\hline No & 98 & 83.1 & 49 & 79.0 & 49 & \\
\hline
\end{tabular}

${ }^{1}$ Calculated by dividing the number of observations by the total number of cases per experimental group. ${ }^{2}$ Comparison between experimental groups was performed using chi-squared analysis or Fisher's exact test.

${ }^{3}$ Cases were enrolled March through December 2015, January through December 2016, and January through May 2017.

${ }^{4}$ Occurrence of diseases (e.g., ketosis, metritis, milk fever, pneumonia, retained placenta) before case detection.

${ }^{5}$ Previous clinical mastitis history within $55 \mathrm{~d}$ before case detection. 
severe $\mathrm{CM}$ on $\mathrm{TF}$ included treatment (forced), parity group, and DIM (Table 4). Compared with cows in the negative control group, IMM treatment did not have an effect on probability of TF $(P=0.236)$, but there were tendencies for parity group and DIM to be associated with this outcome. Odds of TF were 4.4 times greater for the cows in parity $>2$ compared with the younger parity groups. Each 1-d increase in DIM at enrollment increased the probability of TF by $0.6 \%(P=0.055)$.

The power to detect a difference in $\mathrm{TF}$ proportions by experimental group was 0.12 . If the true rate of $\mathrm{TF}$ in the negative control and treated groups was 15.2 and $8.1 \%$ (based on distribution described in Table 3), respectively, 350 cases per experimental group would be required to reject the null hypothesis (power, $P=0.8$ ).

\section{Number Needed to Treat}

Based on least squares means (LSM) of IMI by experimental group (Table 4), 8 quarters would require IMM treatment to prevent 1 IMI $(95 \%$ CI $=6.6-10.0)$. Based on LSM of TF by experimental groups (Table 4), 20 quarters would require IMM treatment to prevent 1
TF $(95 \%$ CI $=12.9-40.6)$. Number needed to treat for QR was not calculated as IMM treatment represented a disadvantage compared with no IMM treatment.

\section{Survival Analysis}

Days to Clinical Cure. Mean days to clinical cure was $4.0 \pm 0.2 \mathrm{~d}$. The probability of clinical cure within $10 \mathrm{~d}$ after enrollment did not vary by season $(P=$ $0.436)$, parity group $(P=0.651)$, CM history $(P=$ $0.322)$, previous disease $(P=0.596)$, severity of $\mathrm{CM}$ $(P=0.739)$, previous SCM $(P=0.243)$, or treatment $(P=0.776$; Figure 2a). Average duration until clinical cure was $4.0 \pm 0.2$ and $4.2 \pm 0.3 \mathrm{~d}$ for cases in the negative control and treated groups, respectively. Of negative control cases, $13.6 \%$ were censored based on no clinical cure $(\mathrm{n}=6)$ or being lost to follow-up $(\mathrm{n}=$ 2 ). Of treated cases, $8.1 \%$ were censored based on no clinical cure $(\mathrm{n}=1)$ or being lost to follow-up $(\mathrm{n}=4)$.

Days to Culling. There were no deaths of cows enrolled in this study. Very few cows $(\mathrm{n}=4$ treated; $\mathrm{n}=2$ negative control) were culled during the FUP (Figure $2 \mathrm{~b}$ ), and mean days to culling was $36.3 \pm 3.2$

Table 3. Univariate associations between selected risk factors and quarter-level recurrence of clinical mastitis (QR; $\mathrm{n}=107)$, IMI at 14 or 28 $\mathrm{d}$ after enrollment $(\mathrm{n}=99)$, and treatment failure $(\mathrm{TF} ; \mathrm{n}=121)$

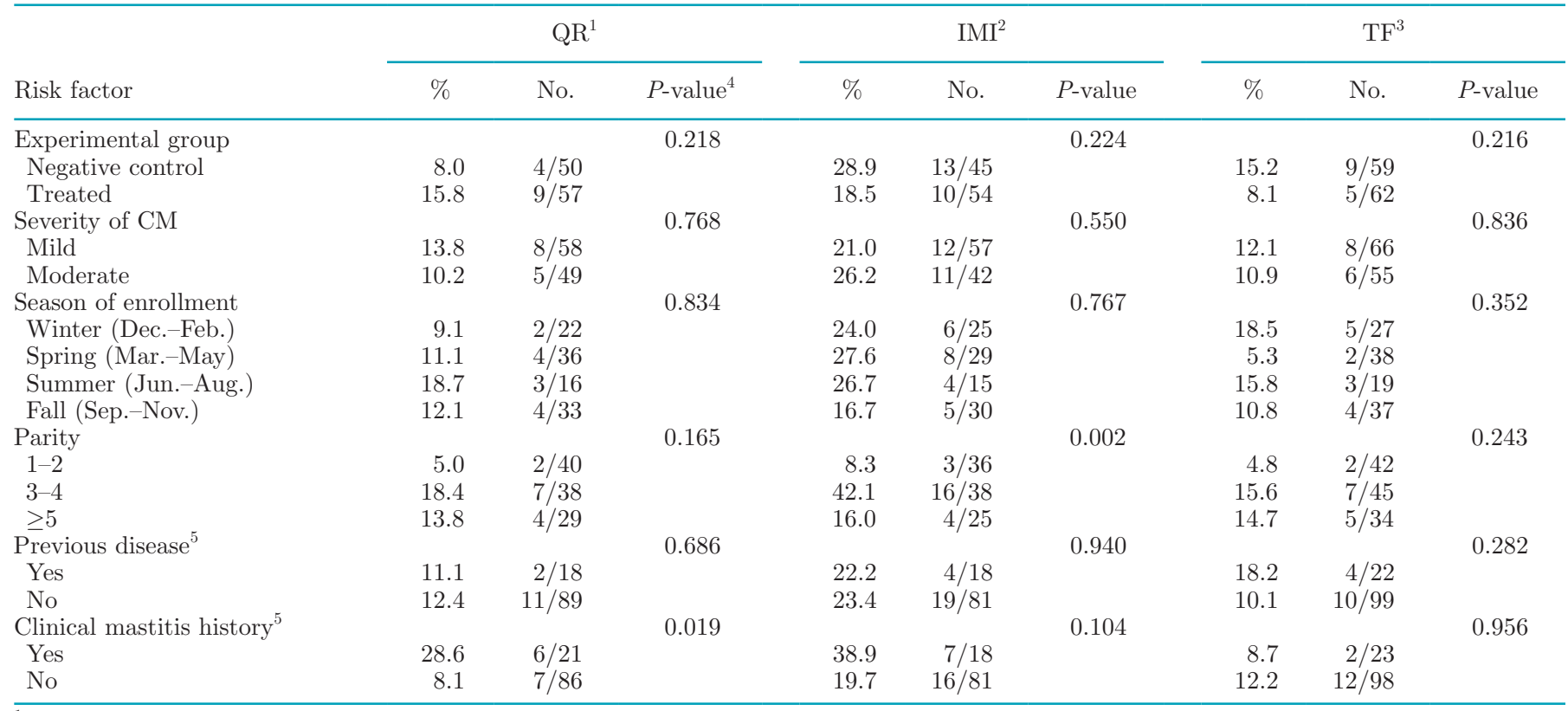

${ }^{1}$ Defined as the detection of a new clinical mastitis (CM) event at least $14 \mathrm{~d}$ after enrollment during a 90-d follow-up period or until dry off. Cases of CM that failed to cure $(\mathrm{n}=14)$ were not included in this analysis.

${ }^{2}$ Defined as bacterial recovery at 14 or $28 \mathrm{~d}$ after enrollment. Intramammary infection was not assessed in 22 cases due to missing samples $(\mathrm{n}=$ $10)$ or contaminated samples $(\mathrm{n}=12)$ at 14 or $28 \mathrm{~d}$ after enrollment.

${ }^{3}$ Defined as administration of a treatment different from the assigned antimicrobial treatment due to worsening severity of CM $(\mathrm{n}=10)$, continued abnormal appearance of milk for $10 \mathrm{~d}$ after enrollment $(\mathrm{n}=3)$, or recurrence of abnormal milk in the same quarter after it had earlier returned to normal (within $14 \mathrm{~d}$ after enrollment; $\mathrm{n}=1$ ).

${ }^{4}$ Comparisons between outcomes and risk factors were performed using chi-squared analysis or Fisher's exact test.

${ }^{5}$ See Table 2 for full definitions. 
Table 4. Final logistic regression models for the effect of intramammary treatment of nonsevere culturenegative clinical mastitis on quarter-level recurrence $(\mathrm{n}=91)$, IMI at 14 or $28 \mathrm{~d}$ after enrollment $(\mathrm{n}=86)$, and treatment failure $(\mathrm{n}=121)$

\begin{tabular}{|c|c|c|c|c|c|}
\hline Outcome & No. & $\operatorname{LSM}^{1}$ & SEM & Odds ratio $(95 \% \mathrm{CI})$ & $P$-value \\
\hline \multicolumn{6}{|l|}{ Quarter-level recurrence $^{2}$} \\
\hline Intercept & & -17.970 & 4.900 & & \\
\hline Experimental group & & & & & 0.524 \\
\hline Negative control & 43 & 0.053 & 0.035 & & \\
\hline Treated & 48 & 0.082 & 0.044 & & \\
\hline Milk yield $7 \mathrm{~d}$ before case detection & 91 & 0.158 & 0.052 & & 0.003 \\
\hline $\log _{10}$ SCC before case detection ${ }^{3}$ & 91 & 1.519 & 0.566 & & 0.009 \\
\hline \multicolumn{6}{|l|}{ IMI at 14 or $28 \mathrm{~d}^{4}$} \\
\hline Intercept & & -7.352 & 2.360 & & \\
\hline Experimental group & & & & & 0.165 \\
\hline Negative control & 40 & 0.259 & 0.079 & & \\
\hline Treated & 46 & 0.133 & 0.053 & & \\
\hline Parity & & & & & 0.016 \\
\hline $1-2$ & 33 & 0.091 & 0.051 & Referent & \\
\hline $3-4$ & 32 & 0.414 & 0.095 & $7.03(1.65-30.02)$ & 0.009 \\
\hline$\geq 5$ & 21 & 0.148 & 0.076 & $1.73(0.31-9.47)$ & 0.520 \\
\hline $\log _{10}$ SCC before case detection & 86 & 1.082 & 0.447 & & 0.018 \\
\hline \multicolumn{6}{|l|}{ Treatment failure } \\
\hline Intercept & & -3.804 & 0.993 & & \\
\hline Experimental group & & & & & 0.236 \\
\hline Negative control & 59 & 0.099 & 0.043 & & \\
\hline Treated & 62 & 0.050 & 0.028 & & \\
\hline Parity & & & & & 0.071 \\
\hline 1 and 2 & 42 & 0.035 & 0.026 & Referent & \\
\hline$>2$ & 79 & 0.138 & 0.041 & $4.39(0.88-21.98)$ & \\
\hline DIM at case detection & 121 & 0.006 & 0.003 & & 0.055 \\
\hline
\end{tabular}

${ }^{1}$ Estimates of predicted probabilities of treatment outcomes were described on the scale of the mean (inverse linked scale).

${ }^{2}$ Cases of clinical mastitis that failed to cure $(\mathrm{n}=14)$ and did not have a monthly DHIA $\log _{10}$ SCC 21 to $55 \mathrm{~d}$ before case detection were not included in this analysis $(\mathrm{n}=16)$.

${ }^{3} \log _{10}$ SCC 21 to 55 d before case detection.

${ }^{4}$ Intramammary infection was not assessed in 22 cases due to missing samples $(\mathrm{n}=10)$ or contaminated samples $(\mathrm{n}=12)$ at 14 or $28 \mathrm{~d}$ after enrollment. Cases of clinical mastitis that did not have a monthly DHIA $\log _{10}$ SCC 21 to $55 \mathrm{~d}$ before case detection were not included in this analysis $(\mathrm{n}=13)$.

d. The probability of remaining in the herd during the FUP was not associated with season $(P=0.878)$, parity group $(P=0.421)$, severity of $\mathrm{CM}(P=0.539)$, previous SCM $(P=0.319)$, CM history $(P=0.859)$, or treatment $(P=0.464)$ but was associated with previous disease $(P=0.001)$. Average duration until cows were culled was $36.8 \pm 0.2$ and $48.3 \pm 0.5 \mathrm{~d}$ for cases in the negative control and treated groups, respectively. After accounting for previous disease $(P=0.009)$, there was no association between treatment and risk of culling $(P$ $=0.522$; Figure 2b).

Days Milk Discarded. Mean days milk discarded was $7.1 \pm 0.3 \mathrm{~d}$. The probability of milk returning to the tank after enrollment did not vary based on parity group $(P=0.203)$, $\mathrm{CM}$ history $(P=0.697)$, previous disease $(P=0.231)$, severity of $\mathrm{CM}(P=0.245)$, or previous SCM $(P=0.864)$ but was associated with treatment $(P<0.001)$ and season of enrollment $(P=$ 0.023; Figure 2c). Average duration of milk out of the tank was $5.6 \pm 0.2$ and $8.5 \pm 0.1 \mathrm{~d}$ for cases in the negative control and treated groups, respectively. Of nontreated cases, $11.9 \%$ were censored based on cases not having the milk returned to the tank $(\mathrm{n}=1)$ or being lost to follow-up $(\mathrm{n}=6)$. Of treated cases, $8.1 \%$ were censored due to being lost to follow-up $(n=5)$.

Days to IMI. Mean days to IMI was $67.6 \pm 3.2$ d. The probability of IMI during the FUP did not vary based on CM history $(P=0.252)$, season of enrollment $(P=0.334)$, previous disease $(P=0.670)$, or severity of CM $(P=0.918)$ but was associated with parity group $(P=0.004)$ and previous SCM $(P=0.011)$. Treatment had a tendency to be associated with the probability of IMI $(P=0.075)$. Of negative control cases, $60.3 \%$ were censored based on cases not developing IMI $(\mathrm{n}=$ 29 ) or being lost to follow-up $(\mathrm{n}=6)$. Of treated cases, $72.1 \%$ were censored based on cases not developing IMI $(\mathrm{n}=39)$ and being lost to follow-up $(\mathrm{n}=5)$. After accounting for parity $(P=0.015)$ and $\log _{10}$ SCC 21 to 
Table 5. Description of bacteria recovered from IMI during the followup period $(7,14,28,63$, and $81 \pm 3 \mathrm{~d}$ after enrollment; $\mathrm{n}=40)$

\begin{tabular}{|c|c|c|c|c|c|}
\hline \multirow[b]{2}{*}{ Pathogen } & \multicolumn{5}{|c|}{ Days after enrollment } \\
\hline & 7 & 14 & 28 & 63 & 81 \\
\hline \multicolumn{6}{|l|}{ Gram positive } \\
\hline Environmental streptococci & 9 & 1 & 1 & 3 & - \\
\hline NAS & 5 & 3 & - & - & - \\
\hline Enterococcus spp. & 1 & 1 & _ & 1 & - \\
\hline Lactococcus spp. & 2 & - & 1 & - & - \\
\hline Trueperella pyogenes & - & 1 & - & - & - \\
\hline Bacillus spp. & - & - & - & 1 & - \\
\hline Corynebacterium spp. & - & - & - & - & 1 \\
\hline \multicolumn{6}{|l|}{ Gram negative } \\
\hline Escherichia coli & 1 & - & - & - & 1 \\
\hline Klebsiella spp. & - & 1 & - & - & - \\
\hline Serratia spp. & - & - & - & - & 1 \\
\hline Pseudomonas spp. & - & 1 & - & - & - \\
\hline Enterobacter spp. & 1 & - & - & - & - \\
\hline $\begin{array}{l}\text { Gram-negative and lactose-negative } \\
\text { rods }\end{array}$ & 1 & - & - & - & - \\
\hline \multicolumn{6}{|l|}{ Other } \\
\hline Mixed infection & _ & - & - & _ & 1 \\
\hline Yeast & 1 & - & - & - & - \\
\hline Total & 21 & 8 & 2 & 5 & 4 \\
\hline
\end{tabular}

$55 \mathrm{~d}$ before case detection $(P=0.032)$, there was an association between treatment and risk of IMI $(P=$ 0.041; Figure 2d).

\section{Weekly QSCC}

During the 90-d FUP, LSM of weekly QSCC was $5.4 \pm 0.1$ and $5.5 \pm 0.1 \log _{10}$ SCC for cases in the negative control and treated groups, respectively, and treatment was not associated with weekly QSCC $(P=$ 0.557 ; Figure 3$)$. Time $(P=0.003)$, parity group $(P$ $=0.003), \mathrm{CM}$ history $(P=0.204)$, previous SCM $(P$ $<0.001)$, occurrence of IMI during FUP $(P<0.001)$, and interaction between treatment and $\mathrm{CM}$ history $(P$ $=0.010)$ were all associated with weekly QSCC. Difference in LSM between younger lactation groups $(<5$ lactations) and older lactation groups ( $\geq 5$ lactations) was about $-0.4 \pm 0.1 \log _{10}$ SCC. Weekly QSCC was greater in cows with CM history $\left(5.6 \pm 0.1 \log _{10} \mathrm{SCC}\right)$ compared with cows with no $\mathrm{CM}$ history $(5.4 \pm 0.1$ $\log _{10}$ SCC) and in cows with previous SCM $(5.7 \pm 0.1$ $\log _{10} \mathrm{SCC}$ ) compared with cows with no previous SCM $\left(5.3 \pm 0.1 \log _{10} \mathrm{SCC}\right)$. Cases that experienced IMI during the FUP had greater QSCC $\left(5.7 \pm 0.1 \log _{10} \mathrm{SCC}\right)$ compared with cases not experiencing IMI $(5.3 \pm 0.1$ $\left.\log _{10} \mathrm{SCC}\right)$.

\section{Daily Milk Production}

During the 90-d FUP, LSM of daily milk production was not associated with daily milk production and was $43.6 \pm 0.3$ and $43.3 \pm 0.3 \mathrm{~kg} /$ cow per day for cases in the negative control and treated groups, respectively $(P=0.340$; Figure 4$)$. Time $(P<0.001)$, parity $(P=$ $0.018)$, season of enrollment $(P<0.001)$, DIM $(P<$ 0.001 ), average daily milk yield 1 to $7 \mathrm{~d}$ before case detection $(P<0.001)$, and the interaction between season of enrollment and parity $(P=0.022)$ were all associated with daily milk production. The younger parity group $(<5$ lactations) produced $42.8 \pm 0.3 \mathrm{~kg} /$ cow per day compared with $44.8 \pm 0.2$ for the older parity group. Daily milk production was greater in cows with cases enrolled during spring $(45.2 \pm 0.3 \mathrm{~kg})$ and summer $(44.3 \pm 0.5 \mathrm{~kg})$ compared with fall $(42.3 \pm 0.3 \mathrm{~kg})$ and winter $(41.9 \pm 0.4 \mathrm{~kg})$.

During the FUP, LSM of daily milk production was $46.3 \pm 0.4$ and $42.7 \pm 0.2 \mathrm{~kg} /$ cow per day in cases that experienced IMI $(\mathrm{n}=35)$ and did not experience IMI $(\mathrm{n}=62 ; P<0.001)$. Time $(P<0.001)$, season of enrollment $(P<0.001)$, DIM $(P<0.001)$, average daily milk yield 1 to $7 \mathrm{~d}$ before case detection $(P<$ $0.001)$, previous SCM $(P=0.023)$, and interaction between IMI and season of enrollment $(P=0.006)$ were all associated with daily milk production. Daily milk production was greater for cows that did not experience previous SCM $(43.5 \pm 0.2 \mathrm{~kg} /$ cow per day) compared with those experiencing previous SCM $(42.5 \pm 0.3 \mathrm{~kg} /$ cow per day).

Table 6. Cross-classification of pathogen groups by previous SCC (cells $/ \mathrm{mL}$ ) recovered from IMI detected at 14 or 28 d after enrollment $(n=86)$

\begin{tabular}{|c|c|c|c|c|c|}
\hline \multirow[b]{2}{*}{ Previous $\mathrm{SCC}^{1}$} & \multicolumn{2}{|c|}{$\mathrm{IMI}^{2}$ no. $(\%)$} & \multirow{2}{*}{$\begin{array}{l}\text { No IMI, } \\
\text { no. }(\%)\end{array}$} & \multirow[b]{2}{*}{ Total } & \multirow[b]{2}{*}{$P$-value ${ }^{2}$} \\
\hline & Gram positive & Gram negative & & & \\
\hline$\leq 150,000$ & $6(33.3)$ & $2(66.7)$ & $46(70.8)$ & 54 & 0.014 \\
\hline$>150,000$ & $12(66.7)$ & $1(33.3)$ & $19(29.2)$ & 32 & \\
\hline Total & 18 & 3 & 65 & 86 & \\
\hline
\end{tabular}

${ }^{1}$ Comparison between IMI categories (i.e., gram positive, gram negative, and no IMI) and category of subclinical mastitis before case detection (21-55 d before case detection) was performed using chi-squared analysis or Fisher's exact test.

${ }^{2}$ See Table 5 for full definitions. This table does not include cases caused by yeast spp. 

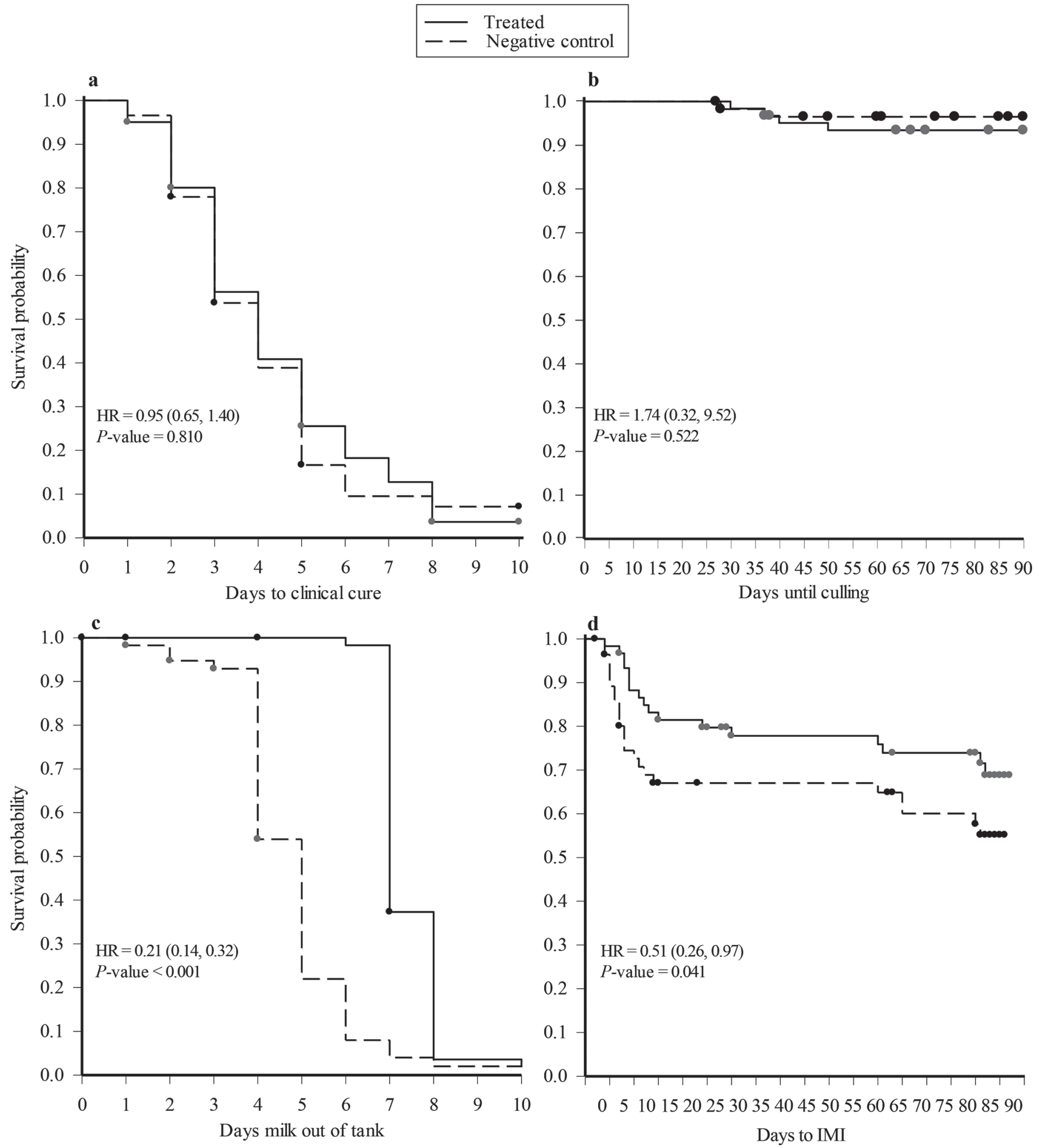

Figure 2. Survival plots describing the probability of (a) clinical cure within $10 \mathrm{~d}$ after enrollment $(\mathrm{n}=121)$, (b) culling $(\mathrm{n}=121)$, (c) discarded milk $(\mathrm{n}=121)$, and $(\mathrm{d})$ IMI $(\mathrm{n}=119)$ by experimental group (negative control and treated). Hazard ratio (HR) between treated and negative control. 


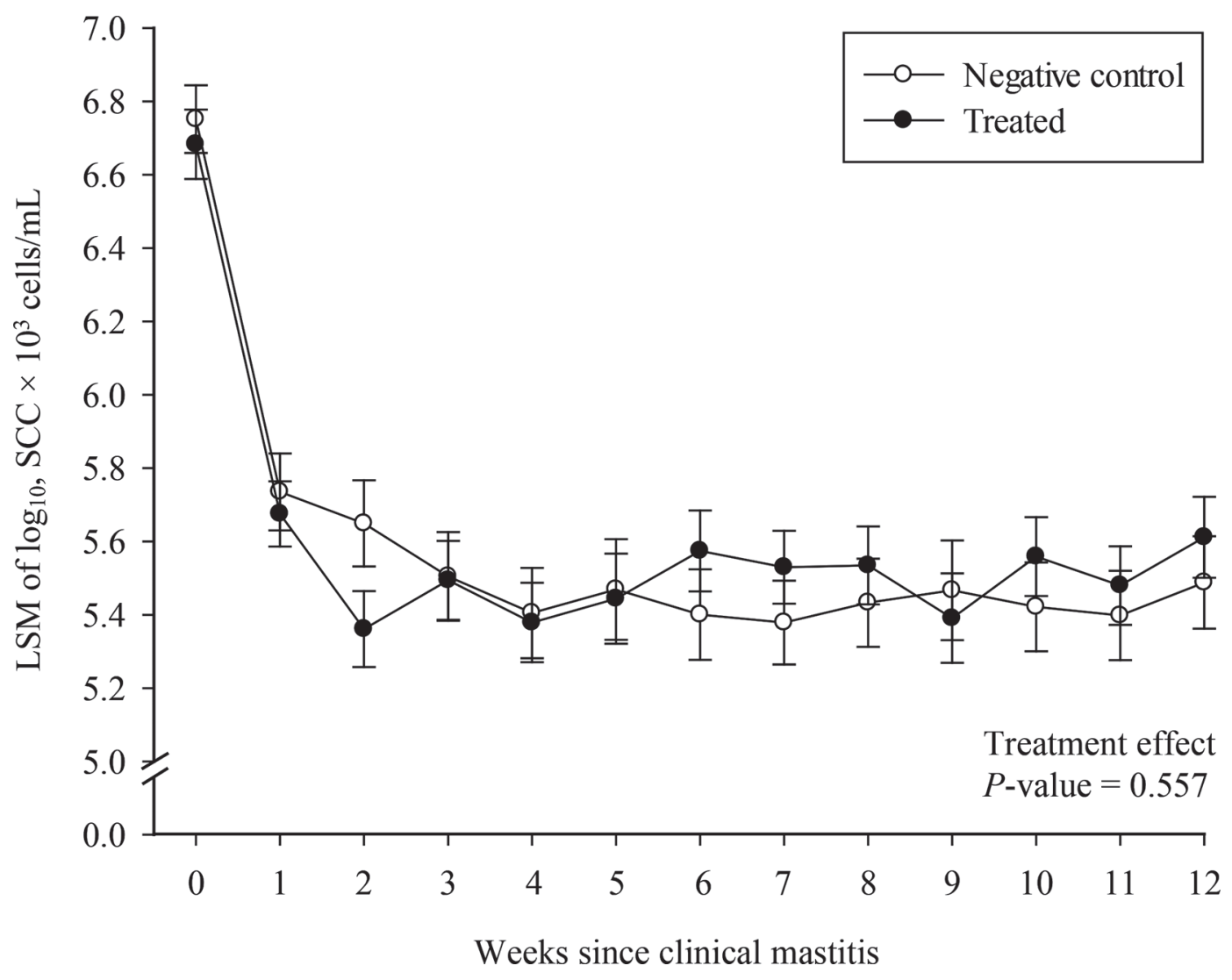

Figure 3. Least squares means of weekly quarter SCC. Negative control group, $\mathrm{n}=42$; treated group, $\mathrm{n}=46$. Repeated-measures analysis included the effect of intramammary treatment, time, parity, previous clinical mastitis, previous subclinical mastitis, occurrence of IMI, and 2-way interactions. Error bars indicate SEM.

\section{DISCUSSION}

Cows in the UW research herd had similar demographic characteristics and were exposed to management practices that were typical of modern conventional dairy farms in the same region (Rowbotham and Ruegg, 2015). Both milk production and SCC of enrolled cows were similar to those of commercial herds located in Wisconsin (Pinzón-Sánchez and Ruegg, 2011; Fuenzalida et al., 2015; Rowbotham and Ruegg, 2015). Compared with previous studies (Oliveira et al., 2013; Fuenzalida, 2015; Ruegg, 2017), a greater proportion of cases of CM was categorized as severe. In a previous study using the same farm, we used the same severity scoring system to classify cows with CM (Fuenzalida, 2015 ); during that study, only $12 \%$ of cases were classified as severe. The greater proportion of severe cases is likely based on enrollment of cows located at a university research farm. These farms are under intense scrutiny by the university animal care and use committee and have strict protocols for animal health. At the beginning of the study, farm workers were concerned about enrolling cows in a study that included a nontreated control group. It is possible that herd managers feared potential consequences of not treating and misclassified nonsevere cases as severe. If this is true, our results may be biased toward cases with less serious clinical signs.

At the beginning of this study, herd managers requested exclusion of potentially eligible cows if milk production at the milking when CM was detected was reduced more than $20 \%$ from the previous daily milking. After 2 mo of enrollment, managers gained experience with the protocol and we were able to remove this criterion. Of 29 nonsevere cases initially excluded due to reduced milk production, only 6 were diagnosed as culture negative and eligible for enrollment; thus, this exclusion had minimal effect on study outcomes. Blinding was not possible because our objective was to contrast outcomes of cows that received IMM treatment with outcomes of cows that were not treated. Use of a placebo would have introduced the potential risk of IMI (Sandholm et al., 1990; Sandgren et al., 2008) and would not be representative of a nontreatment protocol.

Selective treatment is not a new concept, and strategies for managing $\mathrm{CM}$ have been previously described (Lago et al., 2011a,b; Oliveira and Ruegg, 2014; Ruegg, 


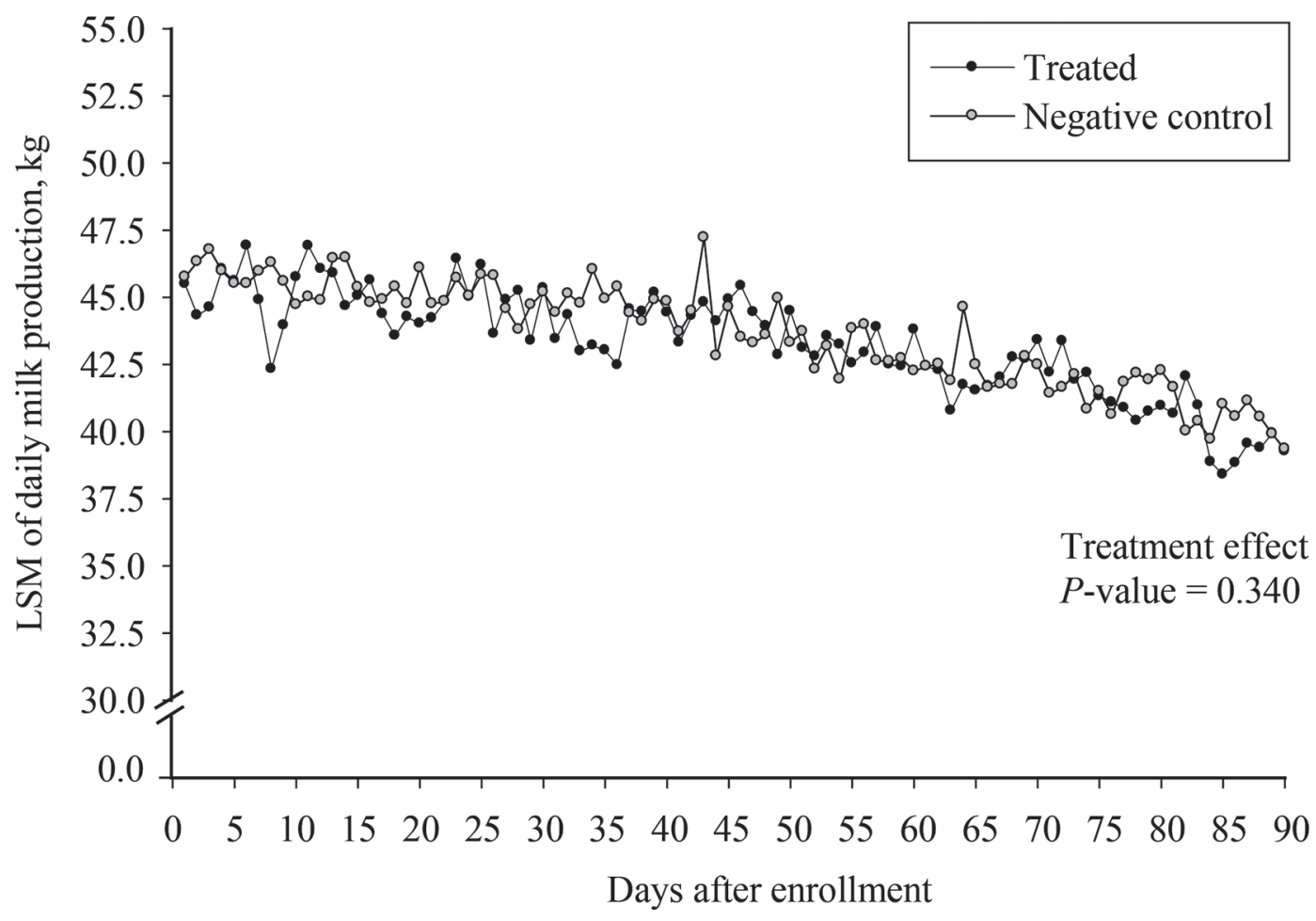

Figure 4. Least squares means of daily milk production. Negative control group, $\mathrm{n}=57$; treated group, $\mathrm{n}=57$. Repeated-measures analysis included the effect of intramammary treatment, time, parity, season of enrollment, DIM, milk production 1 wk before case detection, and 2-way interactions.

2017). Throughout the United States, most cases of CM (including culture-negative cases) are treated without knowledge of etiology (USDA, 2014). Although some farms use culture-based systems to guide therapy, on most large Wisconsin dairy farms, cows with nonsevere CM are treated symptomatically and receive antimicrobials based on severity rather than knowledge of etiology (Oliveira and Ruegg, 2014). Although use of a critically important antimicrobial for treatment of nonsevere CM seems illogical, we selected $5 \mathrm{~d}$ of IMM ceftiofur because this compound is the most frequently used antimicrobial on US farms (USDA, 2014), this protocol is the most common treatment used for nonsevere CM in our region (Oliveira and Ruegg, 2014), and at the beginning of the study this protocol was the standard treatment used on this farm for routine mastitis treatment.

Clinical mastitis is diagnosed based on observation of inflammation of the mammary gland, and many cases are culture negative (Pinzón-Sánchez and Ruegg, 2011; Oliveira et al., 2013; Fuenzalida et al., 2015). The proportion of culture-negative cases was similar to those reported by Pinzón-Sánchez and Ruegg (2011) and Olde Riekerink et al. (2008). Failure to recover pathogens from milk samples collected from cases of CM include spontaneous bacteriological clearance be- fore detection of inflammation (Smith et al., 1985; de Haas et al., 2002), occurrence of false-negative microbiological results (Silva et al., 2005; Lago, 2009), or mastitis caused by a nonculturable bacteria (Metzger, 2018). Occurrence of spontaneous cure is often evident based on rapid resolution of QSCC after resolution of clinical signs; in one study, SCC of culture-negative cases returned to almost-normal values 21 to $28 \mathrm{~d}$ after case detection (de Haas et al., 2002). The probability of spontaneous cure is related to characteristics of both the pathogen and the cow (de Haas et al., 2004). For some pathogens, an effective immune response can successfully eliminate the IMI or rapidly reduce the number of bacterial colonies to a level below normal detection limits. The duration of IMI and rate of spontaneous bacteriological cure are known to be short for $E$. coli and much longer for environmental streptococci (Smith et al., 1985). Although some culture-negative cases are the result of spontaneous bacteriological clearance, some are the result of errors in performing OFC, and others are false-negative cases resulting from collection of a milk sample that contains sparse numbers of bacteria that have been reduced due to prolonged freezing (Schukken et al., 1988; Sears et al., 1990; Silva et al., 2005). However, the OFC were performed using fresh milk, so this possibility was not considered. When 
selective treatment protocols include a no-treatment option, the occurrence of false culture-negative results could lead to retention of subclinically infected cows and to increased risk of recurrence and increased potential for contagious transmission among cows. In these instances, SCC values will remain elevated.

Most cows used in this study were housed in a sandbedded freestall facility; typical of this housing, a large proportion of pathogens cultured from nonsevere CM were gram positive (Rowbotham and Ruegg, 2016). During the enrollment period, the etiology of CM was mainly gram positive as previously described for the same herd (Fuenzalida, 2015). Within gram-positive pathogens, environmental streptococci were the main bacteria recovered from $\mathrm{CM}$ cases. In general, CM caused by environmental streptococci are associated with a longer increase in SCC (de Haas et al., 2004) and longer duration of the disease, with about half of the cases progressing to clinical signs (Smith et al., 1985). During the FUP, we observed that gram-positive bacteria caused the majority of IMI in this herd and that an association existed between SCM and type of pathogens causing IMI.

About $26 \%$ of nontreated culture-negative cases enrolled in our study experienced IMI in contrast to only $13 \%$ of treated cases. Some cases of CM that are diagnosed using OFC as culture negative at $24 \mathrm{~h}$ are false negative, and some of these false negatives were likely diagnosed as experiencing IMI at 7 or $14 \mathrm{~d}$. In our study, most cases of culture-negative $\mathrm{CM}$ that experienced IMI were preceded by high SCC before detection of the original case. This pattern is typical of CM caused by gram-positive bacteria (de Haas et al., 2004) and implies that some of our culture-negative cases may have been false negative. Of culture-positive cases that were not enrolled in our study, the most common result was environmental streptococci, and it is likely that the distribution of false-negative results was similar to this pattern. About $70 \%$ of IMI caused by gram-positive bacteria were associated with previous high SCC, which is more typical of gram-positive than gram-negative pathogens. On a clinical basis, knowledge of the distribution of culture-positive cases in an individual herd and the SCC history of the affected case should be combined with results of OFC to help make informed treatment decisions (Silva et al., 2005; Ruegg, 2017).

Selective treatment protocols have been developed and generally recommend treatment of cases of $\mathrm{CM}$ that are gram positive while withholding antimicrobial treatment from nonsevere cases that are culture negative or gram negative (Lago et al., 2011a,b; Ruegg, 2017). Overall, compared with routine use of antimicrobials for treatment of all cases of CM, selective treat- ment did not affect short- or long-term outcomes and can potentially reduce usage of antimicrobials (Lago et al., 2011a,b). Based on our results, we confirmed that withholding IMM antimicrobial treatment for culturenegative cases of CM did not result in reduced clinical outcomes and was not associated with differences in milk yield or SCC over a 90-d FUP. However, it is important to note that mastitis can be a painful disease and use of nonsteroidal anti-inflammatories that can provide relief of pain should be considered (Leslie and Petersson-Wolfe, 2012). This study was performed in a single herd in North America, and further studies with additional herds and in additional regions are needed to confirm these results.

Several previous researchers have reported outcomes of selective treatment protocols for nonsevere culturenegative CM (Guterbock et al., 1993; Roberson et al., 2004; Pinzón-Sánchez and Ruegg, 2011; Oliveira et al., 2013; Oliveira and Ruegg, 2014); however, only 1 study (Roberson et al., 2004) was designed to contrast IMM therapy with a true negative control group. Results of observational studies have indicated that nontreatment of culture-negative cases resulted in clinical outcomes that are similar to results after antimicrobial treatment (Pinzón-Sánchez and Ruegg, 2011; Oliveira et al., 2013; Oliveira and Ruegg, 2014). Roberson et al. (2004) described that IMM antimicrobial therapy was not efficacious for culture-negative cases based on bacteriological and clinical cure, but only 7 no-growth cases were enrolled in that study. The current study included a true negative control group and administered the most common antimicrobial used for treatment of $\mathrm{CM}$ in the United States. Using a single herd in North America, we were unable to demonstrate that antimicrobial usage was beneficial for treatment of nonsevere culture-negative $\mathrm{CM}$ and did not affect economically important traits such as milk yield, SCC, or culling. Based on typical distributions of culture results from US farms, broader use of nontreatment strategies for culture-negative cases has the potential to reduce usage of antimicrobials by approximately $30 \%$.

Our results confirm our previous studies that indicate clinical cure (return to normal milk) should not be used to evaluate treatment efficacy because the duration until clinical cure does not vary and remains between 4 and 6 d (Hoe and Ruegg, 2005; Pinzón-Sánchez and Ruegg, 2011; Oliveira and Ruegg, 2014). Clinical cure represents the self-limiting inflammatory response within the mammary gland; it does not vary by pathogen (Hoe and Ruegg, 2005; Oliveira and Ruegg, 2014) or treatment (Oliveira and Ruegg, 2014). Results of our study agree with previous reports regarding the length of timing until clinical cure and the low relevance of this outcome to evaluating treatment success of CM. Based 
on our results, farmers should be advised to wait at least $10 \mathrm{~d}$ after detection of a nonsevere culture-negative case (in addition to OFC culture results) before considering treating the same quarter with antimicrobials.

Results of microbiological analysis of milk samples are an objective way to evaluate responses after treatment, but this technique is not practical for the farm setting (Pinzón-Sánchez and Ruegg, 2011). During the FUP, IMI was a frequent event, and we noted that treatment reduced the probability of this outcome. However, most IMI were gram-positive bacteria and were preceded by high SCC, indicating that the culture-negative finding may have been a false negative (potentially caused by gram-positive bacteria). More than $50 \%$ of IMI occurred by $7 \pm 3 \mathrm{~d}$ during the period in which treated cases were still receiving antimicrobials. Oliver et al. (2004b) described that bacteriological cure was significantly greater in cases caused by gram-positive bacteria treated with IMM ceftiofur ( 5 or $8 \mathrm{~d}$ of IMM ceftiofur) compared with no treatment. Despite greater occurrence of IMI for nontreated cases, our results were similar to those of a study that enrolled nontreated, nonsevere gram-negative cases (Schukken et al., 2011) in that we did not identify associations of treatment with economically relevant outcomes such as milk production, culling, or SCC.

In our study, treatment of nonsevere cases of culturenegative $\mathrm{CM}$ did not affect daily milk production during the 90-d FUP. Similar results have been described for cases of CM caused by E. coli (Sandgren et al., 2008; Schukken et al., 2011) and cows randomized to culture-based or standard treatment protocols (Lago et al., 2011b). As expected, we observed that the number of days that milk was discarded was greater for cows that received $5 \mathrm{~d}$ of IMM ceftiofur. Reduced days of discarded milk is an economic driver in support of selective treatment guided by use of OFC. Using a decision tree model, Pinzón-Sánchez et al. (2011) demonstrated that use of culture-based therapy for culture-negative CM resulted in the greatest expected monetary values compared with routine use of antimicrobials. In our study, the milk was discarded $3 \mathrm{~d}$ less for cows in the negative control group. In a dairy herd with lactating cows producing about $44 \mathrm{~kg}$ of milk/d, those $3 \mathrm{~d}$ of milk discarded would have resulted in $132 \mathrm{~kg}$ of milk loss or around $\$ 48$ in losses in economic profit per case (milk price: $\$ 0.36 / \mathrm{kg}$ ). No significant difference in milk yield or SCC was observed during the FUP based on experimental group and few cows were culled, indicating that there is potential to reduce losses due to reduced milk discard when antimicrobials are not administered to nonsevere culture-negative cases.

In this study, QSCC represented the inflammatory response after detection of CM. Our results demonstrated that IMM treatment of nonsevere culture-negative CM cases did not affect weekly QSCC during a 90-d FUP. Similar results have been described in randomized clinical trials that included cases of CM caused by gram-negative (Schukken et al., 2011) or gram-positive (Oliver et al., 2004a) bacteria and for cows randomized to culture-based or standard treatment protocols (Lago et al., 2011b). At enrollment, QSCC remained high, and during the FUP, QSCC decreased rapidly during the first 2 wk of enrollment and remained consistently low afterward. This SCC pattern has been previously described for culture-negative cases of CM (de Haas et al., 2002). From these data, we believed that the immune response of the cow responded quickly to fight an IMI and, as a result, QSCC decreased acutely within a matter of weeks to return to almost normal values. Furthermore, based on QSCC data, most cases of nonsevere culture-negative CM did not benefit from IMM antimicrobial treatment because the immune response of the cow was able to respond effectively. From this point of view, administering IMM antimicrobials for nonsevere culture-negative cases of $\mathrm{CM}$ would only increase cost and would not translate into financial benefits. Farmers might focus resources on preventing new cases of mastitis from occurring or removing cows that are chronically infected from the herd.

Recurrence of CM is an important outcome because it is associated with economically important traits such as reduced sale of milk, increased use of antimicrobials, and increased risk of culling (Pinzón-Sánchez and Ruegg, 2011; Jamali et al., 2018). In our study, we observed a low frequency of $\mathrm{QR}$ in both experimental groups and quarter-level recurrences were similar to those described by Oliveira et al. (2013). Similar to Jamali et al. (2018), both previous milk production and $\log _{10}$ SCC were associated with increased probability of QR. In our study, treatment did not have an effect on QR and was not associated with milk yield. Before initiating antibiotic treatment for $\mathrm{CM}$, herd managers should review the number of recurrent cases at the quarter level so they can identify quarters that are not responding and consider managing those cases using other nonantimicrobial strategies (Ruegg, 2017).

Treatment failure, QR, and culling rarely occurred for cases enrolled in our study. Before initiating the study, we estimated sample size for different outcomes based on data from observational studies (Oliveira et al., 2013) and randomized clinical trials for gramnegative bacteria (Schukken et al., 2011). Based on the small differences that we observed in our groups, we did not have sufficient power to find differences for QR, TF, culling, and IMI at 14 and $28 \mathrm{~d}$ after enrollment. This is likely due to the emphasis on culture-negative cases, which have more positive clinical outcomes com- 
pared with culture-positive cases (Oliveira and Ruegg, 2014; Fuenzalida et al., 2015). Based on the small differences that we observed in binomial outcomes, we would require at least 350 cases per group to identify significance. Studies of this size are logistically and economically infeasible for bovine mastitis and would likely result in detection of small differences that are not biologically or economically relevant. Due to restrictions of this study, larger studies would help confirm our results.

Although we observed some numerical (but not statistically significant) differences in IMI at 14 and $28 \mathrm{~d}$ after enrollment, clinically relevant negative outcomes (QR, culling, and TF) occurred infrequently, and treatment of culture-negative cases would likely have a very limited effect on cow welfare or herd productivity. For example, a 1,000-cow dairy with $25 \%$ incidence of $\mathrm{CM}$ and $30 \%$ rate of culture-negative nonsevere cases would be expected to experience 75 culture-negative cases per year. Ignoring statistical insignificance, based on our univariate proportions, nontreated culture-negative cases would experience $3 \%$ ( 2 cases) fewer recurrences, $13 \%$ (10 cases) more IMI, $5 \%$ (4 cases) more TF, and 3 less days per case $(225 \mathrm{~d})$ of milk discard. Overall, there would be no expected effect on milk yield, culling, or SCC - the most important economic traits in dairy herds. At $44 \mathrm{~kg}$ of milk/d, an additional 9,900 kg of milk would be discarded at a value of about $\$ 3,564(\$ 0.36$ / $\mathrm{kg}$ ), and approximately $\$ 1,688$ would be expended for purchase of IMM antibiotics ( $5 \mathrm{~d}$ for 75 cases $\times \$ 4.50$ / tube), for a total of $\$ 5,252$. These expenditures would occur without any expectation of increased milk yield, reduced SCC, or reduced culling and would result in increased use of critically important antimicrobials.

To understand the effect of treatment decisions for nonsevere culture-negative cases of CM, we calculated the number of cases that need to be treated to prevent 1 adverse outcome. Smaller values indicate greater value for antimicrobial treatment. In our study, 8 cases needed to be treated to prevent 1 IMI, and occurrence of IMI was not associated with negative clinical outcomes. Treatment failure occurred rarely in our study, and 21 cases needed to be treated to prevent $1 \mathrm{TF}$. With these large values, only treatments that resulted in large differences in productivity or longevity would be expected to be cost effective. Given these outcomes, it is difficult to justify use of critically important antimicrobials for treatment of most cases of nonsevere culture-negative CM.

\section{CONCLUSIONS}

Using data from a single herd, IMM treatment with 5 -d ceftiofur therapy did not indicate statistically signif- icant effects on QR, IMI at 14 or 28 d after enrollment, TF, culling, weekly QSCC, or daily milk yield. Treatment had a positive effect on reducing IMI during the entire FUP and a negative effect on the number of days that milk was discarded. The effect of IMM treatment would not be expected to improve any economically relevant clinical outcome such as culling, milk production, or SCC. Negative outcomes such as QR, TF, and culling occurred rarely in nonsevere culture-negative cases of CM. Based on this study, little financial or welfare benefits would be obtained based on use of IMM ceftiofur to treat nonsevere culture-negative CM, but additional studies with larger numbers of animals and conducted in other regions would be useful to confirm these results.

\section{ACKNOWLEDGMENTS}

We thank Cecilia Baumberger (University of Wisconsin, Madison) for helping to conduct this trial and training farm workers on conducting on-farm culture in the university herd. This study was supported by Hatch Act Formula Fund (Wisconsin) grant no. WIS01799.

\section{REFERENCES}

Altman, D. G., and P. K. Andersen. 1999. Calculating the number needed to treat for trials where the outcome is time to an event. BMJ 319:1492-1495.

Andersen, S., I. R. Dohoo, R. Olde Riekerink, H. Stryhn, and Mastitis Research Workers' Conference. 2010. Diagnosing intramammary infections: Evaluating expert opinions on the definition of intramammary infection using conjoint analysis. J. Dairy Sci. 93:2966-2975.

Cohen, J. A. 1960. A coefficient of agreement for nominal scales. Educ. Psychol. Meas. 20:37-46.

de Haas, Y., H. W. Barkema, and R. F. Veerkamp. 2002. The effect of pathogen-specific clinical mastitis on the lactation curve for somatic cell count. J. Dairy Sci. 85:1314-1323.

de Haas, Y., R. F. Veerkamp, H. W. Barkema, Y. T. Gröhn, and Y. H. Schukken. 2004. Associations between pathogen-specific cases of clinical mastitis and somatic cell count patterns. J. Dairy Sci. $87: 95-105$.

Dohoo, I. R.. J. Smith, S. Andersen, D. F. Kelton, S. Godden, and Mastitis Research Workers' Conference. 2011. Diagnosing intramammary infections: Evaluation of definitions based on a single milk sample. J. Dairy Sci. 94:250-261.

Erskine, R. J., R. D. Walker, C. A. Bolin, P. C. Bartlett, and D. G. White. 2002. Trends in antibacterial susceptibility of mastitis pathogens during a seven-year period. J. Dairy Sci. 85:1111-1118.

Fuenzalida, M. J. 2015. The Impact of Mastitis on Fertility in Lactating Dairy Cows. MS Thesis. Department of Dairy Science, University of Wisconsin-Madison.

Fuenzalida, M. J., P. M. Fricke, and P. L. Ruegg. 2015. The association between occurrence and severity of subclinical and clinical mastitis on pregnancies per artificial insemination at first service of Holstein cows. J. Dairy Sci. 98:3791-3805.

Guterbock, W. M., A. L. Van Eenennaam, R. J. Anderson, I. A. Gardner, J. S. Cullor, and C. A. Holmberg. 1993. Efficacy of intramammary antibiotic therapy for treatment of clinical mastitis caused by environmental pathogens. J. Dairy Sci. 76:3437-3444.

Hoe, F. G. H., and P. L. Ruegg. 2005. Relationship between antimicrobial susceptibility of clinical mastitis pathogens and treatment outcomes in cows. J. Am. Vet. Med. Assoc. 227:1461-1468. 
Jamali, H., H. W. Barkema, M. Jacques, E. Lavallée-Bourget, F. Malouin, V. Saini, H. Stryhn, and S. Dufour. 2018. Invited review: Incidence, risk factors, and effects of clinical mastitis recurrence in dairy cows. J. Dairy Sci. 101:4729-4746.

Lago, A., S. M. Godden, R. Bey, P. L. Ruegg, and K. Leslie. 2011a. The selective treatment of clinical mastitis based on on-farm culture results: I. Effects on antibiotic use, milk withholding time, and short-term clinical and bacteriological outcomes. J. Dairy Sci. 94:4441-4456.

Lago, A., S. M. Godden, R. Bey, P. L. Ruegg, and K. Leslie. 2011b. The selective treatment of clinical mastitis based on on-farm culture results: II. Effects on lactation performance, including clinical mastitis recurrence, somatic cell count, milk production, and cow survival. J. Dairy Sci. 94:4457-4467.

Lago, J. A. 2009. Efficacy of On-Farm Programs for the Diagnosis and Selective Treatment of Clinical and Subclinical Mastitis in Dairy Cattle. PhD Diss. University of Minnesota, St. Paul.

Lavon, Y., E. Ezra, G. Leitner, and D. Wolfenson. 2011. Association of conception rate with pattern and level of somatic cell count elevation relative to time of insemination in dairy cows. J. Dairy Sci. 94:4538-4545.

Leslie, K. E., and C. S. Petersson-Wolfe. 2012. Assessment and management of pain in dairy cows with clinical mastitis. Vet. Clin. North Am. Food Anim. Pract. 28:289-305.

Makovec, J. A., and P. L. Ruegg. 2003a. Antimicrobial resistance of bacteria isolated from dairy cow milk samples submitted for bacterial culture: 8,905 samples (1994-2001). J. Am. Vet. Med. Assoc. $222: 1582-1589$.

Makovec, J. A., and P. L. Ruegg. 2003b. Results of milk samples submitted for microbiological examination in Wisconsin from 1994 to 2001. J. Dairy Sci. 86:3466-3472.

Mansion-de Vries, E. M., J. Lucking, N. Wente, C. Zinke, M. Hoedemaker, and V. Kromker. 2016. Comparison of an evidence-based and a conventional mastitis therapy concept with regard to cure rates and antibiotic usage. Milchwissenschaft 69:23-26.

Metzger, S. A. 2018. The Milk Microbiota of Healthy and Inflamed Bovine Mammary Glands. PhD Diss. University of WisconsinMadison.

National Mastitis Council. 1999. Laboratory Handbook on Bovine Mastitis. National Mastitis Council, Verona, WI.

National Mastitis Council. 2017. Laboratory Handbook on Bovine Mastitis. 3rd ed. National Mastitis Council, Verona, WI.

Olde Riekerink, R. G. M., H. W. Barkema, D. F. Kelton, and D. T. Scholl. 2008. Incidence rate of clinical mastitis on Canadian dairy farms. J. Dairy Sci. 91:1366-1377.

Oliveira, L., C. Hulland, and P. L. Ruegg. 2013. Characterization of clinical mastitis occurring in cows on 50 large dairy herds in Wisconsin. J. Dairy Sci. 96:7538-7549.

Oliveira, L., and P. L. Ruegg. 2014. Treatments of clinical mastitis occurring in cows on 51 large dairy herds in Wisconsin. J. Dairy Sci. 97:5426-5436.

Oliver, S. P., R. A. Almeida, B. E. Gillespie, S. J. Headrick, H. H. Dowlen, D. L. Johnson, K. C. Lamar, S. T. Chester, and W. M. Moseley. 2004a. Extended ceftiofur therapy for treatment of experimentally-induced Streptococcus uberis mastitis in lactating dairy cattle. J. Dairy Sci. 87:3322-3329.

Oliver, S. P., B. E. Gillespie, S. J. Headrick, H. Moorehead, P. Lunn, H. H. Dowlen, D. L. Johnson, K. C. Lamar, S. T. Chester, and W. M. Moseley. 2004b. Efficacy of extended ceftiofur intramammary therapy for treatment of subclinical mastitis in lactating dairy cows. J. Dairy Sci. 87:2393-2400.

Pinzón-Sánchez, C., V. E. Cabrera, and P. L. Ruegg. 2011. Decision tree analysis of treatment strategies for mild and moderate cases of clinical mastitis occurring in early lactation. J. Dairy Sci. 94:1873-1892.

Pinzón-Sánchez, C., and P. L. Ruegg. 2011. Risk factors associated with short-term post-treatment outcomes of clinical mastitis. J. Dairy Sci. 94:3397-3410.

Pyörälä, S., L. Kaartinen, H. Käck, and V. Rainio. 1994. Efficacy of two therapy regimens for treatment of experimentally induced Escherichia coli mastitis in cows. J. Dairy Sci. 77:453-461.
Pyörälä, S. H. K., and E. O. Pyörälä. 1998. Efficacy of parenteral administration of three antimicrobial agents in treatment of clinical mastitis in lactating cows: 487 cases (1989-1995). J. Am. Vet. Med. Assoc. 212:407-412.

Roberson, J. R., L. D. Warnick, and G. Moore. 2004. Mild to moderate clinical mastitis: Efficacy of intramammary amoxicillin, frequent milk-out, a combined intramammary amoxicillin, and frequent milk-out treatment versus no treatment. J. Dairy Sci. 87:583-592.

Rowbotham, R. F., and P. L. Ruegg. 2015. Association of bedding types with management practices and indicators of milk quality on larger Wisconsin dairy farms. J. Dairy Sci. 98:7865-7885.

Rowbotham, R. F., and P. L. Ruegg. 2016. Association of selected bedding types with incidence rates of subclinical and clinical mastitis in primiparous Holstein dairy cows. J. Dairy Sci. 99:4707-4717.

Ruegg, P. L. 2017. Practical approaches to mastitis therapy on large dairy herds. Pages 933-948 in Large Dairy Herd Management. 3rd ed. D. K. Beede, ed. American Dairy Science Association, Champaign, IL.

Sandgren, C. H., K. P. Waller, and U. Emanuelson. 2008. Therapeutic effects of systemic or intramammary antimicrobial treatment of bovine subclinical mastitis during lactation. Vet. J. 175:108-117.

Sandholm, M., L. Kaartinen, and S. Pyörälä. 1990. Bovine mastitisWhy does antibiotic therapy not always work? An overview. J. Vet. Phamacol. Ther. 13:248-260.

SAS Institute. 2011. SAS/STAT User's Guide. Version 9.4. SAS Institute Inc., Cary, NC.

Schukken, Y. H., G. J. Bennett, M. J. Zurakowski, H. L. Sharkey B. J. Rauch, M. J. Thomas, B. Ceglowski, R. L. Saltman, N. Belomestnykh, and R. N. Zadoks. 2011. Randomized clinical trial to evaluate the efficacy of 5-day ceftiofur hydrochloride intramammary treatment on nonsevere gram-negative clinical mastitis. J. Dairy Sci. 94:6203-6215.

Schukken, Y. H., J. A. H. Smit, F. J. Grommers, D. Vandegeer, and A. Brand. 1989. Effect of freezing on bacteriologic culturing of mastitis milk samples. J. Dairy Sci. 72:1900-1906.

Sears, P. M., B. S. Smith, P. B. English, P. S. Herer, and R. N. Gonzalez. 1990. Shedding pattern of Staphylococcus aureus from bovine intramammary infections. J. Dairy Sci. 73:2785-2789.

Sérieys, F., Y. Raguet, L. Goby, H. Schmidt, and G. Friton. 2005. Comparative efficacy of local and systemic antibiotic treatment in lactating cows with clinical mastitis. J. Dairy Sci. 88:93-99.

Sifferlin, A. 2017. Here's how to cut antibiotic use in animals. Accessed May 2, 2018. http://time.com/4961051/antibiotics-animals/.

Silva, B. O., D. Z. Caraviello, A. C. Rodrigues, and P. L. Ruegg. 2005. Evaluation of petrifilm for the isolation of Staphylococcus aureus from milk samples. J. Dairy Sci. 88:3000-3008.

Smith, K. L., D. A. Todhunter, and P. S. Schoenberger. 1985. Environmental pathogens and intramammary infection during the dry period. J. Dairy Sci. 68:402-417.

Swinkels, J. M., V. Krömker, and T. J. Lam. 2014. Efficacy of standard vs. extended intramammary cefquinome treatment of clinical mastitis in cows with persistent high somatic cell counts. J. Dairy Res. 81:424-433.

Swinkels, J. M., T. J. Lam, M. J. Green, and A. J. Bradley. 2013. Effect of extended cefquinome treatment on clinical persistence or recurrence of environmental clinical mastitis. Vet. J. 197:682-687.

University of Minnesota Laboratory for Udder Health. 2004. Minnesota Easy Culture System II User's Manual. University of Minnesota, St. Paul.

USDA. 2014. Milk quality, milking procedures, and mastitis on U.S. dairies, 2014. Accessed Apr. 2, 2018. https://www.aphis.usda.gov/ animal_health/nahms/dairy/downloads/dairy14/Dairy14_dr _Mastitis.pdf.

Vasquez, A. K., D. V. Nydam, M. B. Capel, S. Eicker, and P. D. Virkler. 2017. Clinical outcome comparison of immediate blanket treatment versus a delayed pathogen-based treatment protocol for clinical mastitis in a New York dairy herd. J. Dairy Sci. 100:2992-3003. 